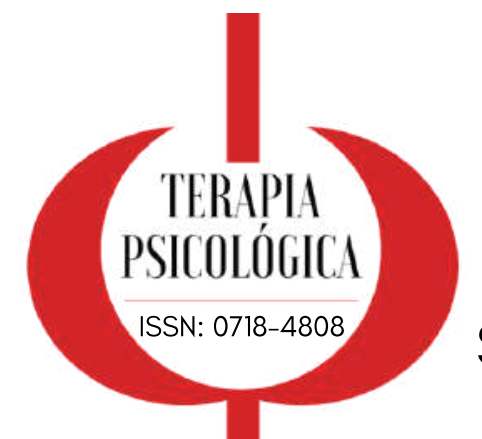

\title{
Riesgo suicida y su relación con la inteligencia emocional y la autoestima en estudiantes universitarios
}

\section{Suicidal risk and its relation with emotional intelligence and self-esteem in university students}

Anyerson Stiths Gómez Tabares

(iD) 0000-0001-7389-3178

anyerspn.gomezta@amigo.edu.co

Universidad Católica Luis Amigó.

Medellín, Colombia.

Programa de Psicología.

\section{María Paula Agudelo Osorio \\ (iD) 0000-0003-3895-8380 \\ magudelo@soyudemedellin.edu.co \\ Universidad de Medellín. \\ Medellín, Colombia. \\ Grupo de Investigación en Psicología y Procesos \\ Clínico-Sociales.Programa de Psicología.}

\author{
César Núñez \\ (iD) 0000-0001-8925-993X \\ cnunez@udem.edu.co \\ Universidad de Medellín. \\ Medellín, Colombia. \\ Programa de Psicología. \\ Vicente E. Caballo \\ 0000-0002-2767-8028 \\ vcaballo@ugr.es \\ Universidad de Granada. \\ Granada, España. \\ Facultad de Psicología.
}

\section{Resumen:}

El objetivo fue establecer la relación del riesgo suicida con la inteligencia emocional y autoestima en una muestra de 1414 estudiantes universitarios de dos ciudades colombianas a partir de un estudio cuantitativo, con diseño no experimental de tipo transversal. Se usaron como instrumentos la Escala de Riesgo Suicida de Plutchik (RS), las versiones adaptadas de Trait Meta-Mood Scale (TMMS-24) y la Escala de Autoestima de Rosenberg (RAE), y una Ficha sociodemográfica ad hoc. Los resultados evidencian correlaciones estadísticamente significativas ( $p<.001)$ positivas entre riesgo suicida, atención emocional y autodesprecio; y negativas $(p<.001)$ entre riesgo suicida, inteligencia emocional, claridad emocional, regulación emocional, autoestima y autoconfianza. Se indica que la inteligencia emocional respecto de la claridad, regulación, autoestima y autoconfianza son factores protectores del riesgo suicida, $y$, al contrario, la atención emocional y el autodesprecio son factores de riesgo para el suicidio.

Palabras clave: riesgo suicida; inteligencia emocional; autoestima; estudiantes universitarios.

\section{Abstract:}

The objective was to establish of suicidal risk with emotional intelligence and self-esteem in a sample of 1414 university students of two Colombian cities from a quantitative study, with a non-experimental crosssectional design. The Plutchik-RS Suicide Risk Scale, the adapted versions of the Trait Meta-Mood Scale (TMMS-24) and the Rosenberg-RAE Scale of Self-Esteem and an ad hoc Sociodemographic Record was used as instruments. The results show positive statistically significant correlations ( $p>0.001)$ among suicidal risk, emotional attention and self-deprecation; and negative correlations ( $p>0.001$ ) among suicidal risk, emotional intelligence, emotional clarity, emotional regulation, self-esteem and self-confidence. We would like to highlight that emotional intelligence regarding clarity, regulation, self-esteem and self-confidence are protective factors of suicidal risk, and conversely, emotional attention and self-deprecation are risk factors for suicide.

Keywords: suicidal risk; emotional intelligence; self-esteem; university students. 


\section{Introducción}

El comportamiento suicida es una problemática de salud pública que ha ido aumentando a través del tiempo. A nivel mundial se estima que aproximadamente 800.000 personas se suicidan cada año, representando el $1,4 \%$ de la morbilidad de años de vida ajustado a la discapacidad, y se prevé que para el 2020 esta cifra pueda incrementarse a 1.500 .000 personas (WHO, 2018), aspecto altamente probable en estudiantes universitarios al relacionarse con los efectos psicológicos en la salud mental con la pandemia COVID-19 (Patsali et al., 2020).

El suicidio se constituye en la segunda causa de muerte en jóvenes entre 15 y los 29 años (WHO, 2018), y el 79\% de todos los suicidios se produce en países de ingresos bajos y medianos y los métodos mantienen una relación estándar con la ingestión de plaguicidas, el ahorcamiento y las armas de fuego (OMS, 2019); actualmente se perfila el nivel de letalidad de la conducta suicida en adolescentes asociado a un historial de intento de suicidio y suicidio más proximal (Rengasamy et al., 2020).

Igualmente, se muestra que por cada suicidio hay muchas más tentativas de suicidio anuales que no son registradas, por lo que es importante considerar que un intento de suicidio no consumado es el factor individual de riesgo más importante, pues un tercio de las personas con ideación suicida pasan al intento (Kleiman \& Liu, 2013); por cada suicidio consumado hay aproximadamente entre 13 a 20 personas que lo intentan (Gómez et al., 2020). En Colombia, de acuerdo con el boletín del Instituto de Medicina Legal y Ciencias Forenses (2020), hasta agosto de 2020 se presentaron un total de 1531 suicidios, y se mantiene la misma tendencia de edad entre 15 y 29 años.

El estigma social frente al suicidio puede interferir con el registro correcto de este fenómeno (WHO, 2014; Villalobos, 2009); se ha demostrado históricamente una asociación con el miedo a la desaprobación social y las objeciones morales frente al acto de suicidarse (Linehan \& Goodstein, 1983). Al respecto, hay evidencia que muestra que, al número de intentos de suicidio a lo largo de la vida y tiempo transcurrido desde el intento de suicidio más reciente, y el estigma de suicidio anticipado (percibir al otro como suicida) se asoció significativamente con un aumento de las tendencias suicidas y mostró un vínculo estadístico parcialmente mediado por un mayor secreto guardado en los suicidas (Mayer et al., 2020). 
Ahora bien, gran parte de la investigación psicológica ha girado en torno al estudio de los factores de riesgo asociados a la conducta suicida, pero se muestra escasa la literatura sobre inteligencia emocional y la autoestima como variables mediadora de la salud mental y riesgo suicida en población universitaria, y más bien si, a definir cómo los recursos personales ayudan a las estrategias preventivas y disuasivas del riesgo suicida (Anton et al., 2013; Mamani et al., 2018), lo que implica reconocer la valía personal en el enfrentamiento individual (Sánchez et al., 2015) y el soporte social protector (Zadravec et al., 2017), lo mismo que condiciones de contexto. De hecho, se han hecho contrastes respecto a variables psicosociales que reportan condiciones de equivalencia del riesgo de muerte por suicidio en jóvenes respecto de la muerte por violencia ubicados como factores de riesgo comunes y específicos (Werbart et al., 2020).

El presente estudio se centra en la relación de la inteligencia emocional y la autoestima con el riesgo suicida en estudiantes universitarios. La inteligencia emocional se define como un conjunto de habilidades adaptativas que tiene una persona y que le permiten percibir, asimilar, comprender y regular las emociones con fines adaptativos (Salovey \& Grewal, 2006). Ello se asocia especialmente con percibir los propios sentimientos y los de otros, la habilidad para identificar la manera en que los sentimientos afectan la capacidad para pensar y resolver problemas (Núñez et al., 2002), lo mismo que discriminar las emociones y sentimientos útiles en las propias acciones de educación emocional (Fernández \& Extremera, 2005).

El concepto de inteligencia emocional fue usado por primera vez por Salovey y Mayer (1990) como un tipo de inteligencia social, que involucra la habilidad de controlar las propias emociones y las de los demás, lo mismo que discriminar entre ellas y orientar el pensamiento hacia acciones del valor mutuo. Incluye la evaluación verbal y no verbal, la expresión emocional, la regulación de la emoción propia y en otros casos y la utilización del contenido emocional para resolver problemas (Mayer \& Salovey, 1993). Históricamente Salovey y Mayer (1990) retoman las inteligencias personales de Gardner (1983) y las ubican en cinco dominios principales: conocer las propias emociones, el manejo de las emociones, el motivarse a uno mismo, el reconocer las emociones en los demás y la capacidad de relacionarse con los demás.

Distintas investigaciones concuerdan en que la inteligencia emocional es un factor protector importante para el riesgo suicida (Abdollahi et al., 2016; Gómez et al., 2018, Mérida-López et al., 2018), pero no se especifica su relación con la población del presente estudio, ni tampoco con la variable autoestima. Se han realizado asociaciones de la inteligencia emocional y riesgo suicida con otras variables como optimismo y esperanza, estilos atribucionales positivos, 
control de impulsos y empatía (Chang et al., 2017; Suárez et al., 2016). La inteligencia emocional se asocia con la claridad y regulación emocional, aspecto que precisamente es carente en personas con pensamientos y comportamientos suicidas (Cha \& Nock, 2009), a quienes se les dificulta gestionar emociones negativas.

Por otro lado, y en contexto del estudio, la autoestima implica la actitud y evaluación que una persona hace de su propio valor e importancia (Atienza et al., 2000) y abarca la capacidad para reconocer y aceptar los propias habilidades, conocimientos y características corporales (Aydin et al., 2018).

La autoestima es un factor protector relevante en la comprensión del riesgo suicida y de cualquier conducta autolesiva (González- Fuentes \& Andrade, 2013; Rasmussen et al., 2015) y tiene efectos similares en distintas condiciones geográficas (Chatard et al., 2009), al mostrar que personas con intentos de suicidio tienen un menor nivel de autoestima en relación con personas sin intentos previos.

A nivel universitario, la relación entre autoestima e inteligencia emocional y riesgo suicida se ha estudiado respecto de la regulación emocional (Quintana et al., 2020), empatía (Sa et al., 2019), ajuste contextual y emocional a condiciones específicas universitarias (Bruns \& Letcher, 2018; Domínguez et al., 2015; Forrester et al., 2017).

De acuerdo con lo planteado hasta el momento y por la necesidad de generar explicaciones de la asociación entre inteligencia emocional y la autoestima frente al riesgo suicida en estudiantes universitarios, se tuvo como objetivo identificar la relación y asociación de dichas variables con una muestra de estudiantes universitarios de dos ciudades colombianas, Manizales y Medellín.

En este sentido, la hipótesis central es que dichas variables se asocian con el riesgo de suicidio en universitarios, pero resultó también novedoso considerar los efectos indirectos de las variables, aspectos que se ubican claramente en el método estadístico de análisis.

Con este estudio se espera contribuir al desarrollo nuevos avances en la comprensión del riesgo suicida en universitarios y entre tanto a su prevención, con una clara intención de ser considerado bajo una perspectiva de atención orientada desde modelos conceptuales de sistemas articulados de bienestar universitario (Núñez et al., 2006). 


\section{Método}

\section{Participantes}

A partir de un diseño no experimental de corte transversal (Hernández et al., 2014) y con un alcance correlacional se tuvo la finalidad de conocer la relación o grado de asociación entre un grupo de variables en un contexto especifico, para lo cual se utilizó un muestreo probabilístico aleatorio estratificado por semestres compuesto de 1414 estudiantes universitarios pertenecientes a centros universitarios de las ciudades de Manizales ( $n=762$; $53.9 \%$ ), y Medellín ( $n=652 ; 46.1 \%$ ). En relación con el sexo, 968 fueron mujeres $(68.5 \%$ ) y 446 hombres (31.5\%). La edad media fue de 20.49 (DE=3.28). Las edades comprendidas fueron de los 16 a los 30 años, distribuidos por rangos: de 16 a 20 años (n=872; 61.7\%), 21 a 25 años $(n=407 ; 28.8 \%), 26$ a 30 años $(n=135 ; 9.5 \%)$.

\section{Instrumentos}

Ficha sociodemográfica: Se aplicó una ficha sociodemográfica elaborada por los investigadores y aprobada por expertos, con información acerca de la edad, el sexo, universidad, semestre académico, jornada, estrato socioeconómico, antecedentes individuales y familiares de conducta suicida.

Escala de riesgo suicida de Plutchik- RS. Es una escala tipo Likert diseñada por Plutchik (Plutchik \& Van Praag, 1989), para evaluar el nivel de riesgo para un intento suicida, así como sentimientos relacionados con la depresión y la desesperanza. Consta de 15 reactivos cuyas opciones de respuesta son de $\mathrm{Si}$ o No, cada respuesta afirmativa suma un punto, para un total de 15. La validación española presenta una consistencia de interna de 0.90 , fiabilidad tesretest de 0.89 , sensibilidad y especificidad de $88 \%$ para un punto de corte en 6 (Rubio, et al., 1998). Ha sido validado en la población colombiana por Ramírez \& Olivella (2014) con una fiabilidad con Alpha de Cronbach de .77. En Colombia se ha utilizado con población de adolescentes y jóvenes (Cañon et al, 2012), mostrando una buena consistencia interna. El análisis de fiabilidad con Alpha de Cronbach para el estudio mostró un coeficiente de .75.

Escala Rasgo de Meta-Conocimiento emocional o Trait Meta-Mood Scale-24 (TMMS-24). Diseñada por Salovey et al. (1995) para evaluar la inteligencia emocional percibida. Se utilizó la adaptación en español de la versión reducida de 24 ítems, la cual ha evidenciado una consistencia interna superior a .80. (Fernández-Berrocal et al., 2004). La TMMS-24 evalúa tres dimensiones de 8 ítems cada una: atención emocional (creencias o percepciones que tienen las personas sobre su capacidad de atención y valoración de sus sentimientos), clari- 
dad emocional (comprensión y análisis de las propias emociones y relaciones entre ellas) y regulación emocional (habilidad para regular o capacidad de autoeficacia sobre las propias emociones) (Gómez et al., 2018). Para determinar la fiabilidad de las dimensiones se utilizó el omega de Mcdonald, el cual arrojó un coeficiente de .91 para atención emocional, .93 para claridad emocional y .91 para regulación emocional. El Alpha de Cronbach para el constructo total arrojó un coeficiente de .91.

Escala de autoestima de Rosenberg (1965- RAE). Escala ampliamente utilizada alrededor del mundo, y traducida a más de 20 idiomas para evaluar la percepción de autoestima y valía personal en diversos grupos poblacionales. Se utilizó la versión adaptada al español utilizada y validada en diversos estudios con población colombiana (Ceballos et al., 2015; Cogollo et al., 2015; Gómez-Lugo et al., 2016) evidenciado una fiabilidad entre .72 y .86. Consta de 10 preguntas tipo Likert de cuatro opciones de respuesta ( 1 "muy en desacuerdo" a 4 "muy de acuerdo"). Para la calificación de la prueba se puede adoptar dos modelos. El primero es unifactorial, el cual mide la autoestima global; el segundo es bifactorial, compuesto de las variables de autoconfianza y autodesprecio. Para este estudio se utilizó el modelo bifactorial. Su calificación total oscila en un rango de 10 (baja autoestima) a 40 puntos (alta autoestima). El análisis de fiabilidad con omega de Mcdonald arrojó un coeficiente de .88 para autoconfianza y .83 para autodesprecio. El Alpha de Cronbach para la escala global fue de .84 .

\section{Consideraciones éticas}

En consideración con ley 1090 de 2006 y la resolución 008430 de 1993, esta investigación tuvo en cuenta los principios éticos de respeto, intimidad y dignidad, asegurando la confidencialidad y el anonimato de los participantes, tal y como se establece en el artículo 26 y 50. Se contó con el aval del comité de ética de la Universidad Católica Luis Amigó para la aplicación de los instrumentos, y el consentimiento informado de los participantes. El proceso de investigación contempló el proceso de remisión al área de psicología de bienestar universitario a los estudiantes identificados en riesgo, tras la calificación manual de los instrumentos posterior a su aplicación.

\section{Procedimiento de análisis de datos}

Los resultados de la aplicación de los instrumentos de medición fueron digitalizados y codificados en una matriz de datos de Excel. Para el análisis estadístico se utilizó el paquete estadístico SPSS versión 25.0 (IBM Corporation, 2017). Inicialmente se realizó la descripción sociodemográfica de la muestra, seguido de un análisis de consistencia interna de las escalas 
y subescalas de los instrumentos. Para las escalas totales se utilizó el coeficiente de alfa de Cronbach y para las subescalas el coeficiente Omega ( $\omega$ ) de McDonald (1999). El coeficiente omega, a diferencia del coeficiente de alfa trabaja con las cargas factoriales (Gerbing \& Anderson, 1988), esto permite mayor estabilidad en los cálculos para escalas multidimensionales, dado que no depende del número de ítems (McDonald, 1999). Se realizó un análisis descriptivo y de frecuencia respecto al factor de riesgo, antecedentes familiares e individuales de intento de suicidio. Se verifico la normalidad de los datos mediante la prueba de Kolmogorov-Smirnov, la cual arrojó que las variables no seguían una distribución normal, motivo por el cual se utilizó la prueba no paramétrica $U$ de Mann - Whitney para realizar un análisis comparativo del riesgo suicida en función de las variables de inteligencia emocional y autoestima. Se utilizó R Studio Cloud para calcular el tamaño del efecto de las diferencias encontradas en el análisis comparativo, el cual se estimó mediante el estadístico de eta cuadrado $\left(\eta^{2}\right)$. Se siguió el procedimiento e interpretación establecido por Fritz et al. (2012).

Posteriormente se realizó un análisis de correlación utilizando el coeficiente rho de Sperman y se procedió a un análisis de regresión logística binaria para identificar las variables de mayor valor predictor sobre el riesgo suicida. Se realizó un análisis de regresión logística binaria, mediante el método de introducción, con el fin de identificar el efecto de la inteligencia emocional y la autoestima sobre el factor de riesgo suicida. Finalmente, se propone un modelo de ecuaciones estructurales para determinar el efecto total, directo e indirecto entre las variables consideradas en este estudio. Para el modelado de ecuaciones estructurales se utilizó el software Amos versión 24.0 (IBM Corporation, 2017). Los efectos totales, directos e indirectos se calcularon utilizando el método bootstrap con un intervalo de confianza del $95 \%$ (Byrne, 2016; Hayes, 2018). Para evaluar la bondad de ajuste de los modelos, se utilizó los valores de chi cuadrado $(X)$, el nivel de probabilidad $(p \geqslant 0,05)$, índices de ajuste comparativo ( $I F I \geqslant 0,90$ y $C F I \geqslant 0,90)$, índice de bondad de ajuste $(G F I \geqslant 0,90)$ y su correspondiente corregido $(\mathrm{AGFI} \geqslant 0,90)$, el índice de ajuste normalizado $(\mathrm{NFI} \geqslant 0,90)$ y el índice de Tucker - Lewis $(T L I \geqslant 0,90)$ y la raíz del residuo cuadrático promedio de aproximación (RMSA $\leqslant 0,08)$ (Byrne, 2016; McArdle \& Nesselroade, 2014).

\section{Resultados}

De acuerdo con los criterios establecidos de la escala de Plutchik, tomando un punto de corte de 6, se identificó que, de los 1414 estudiantes universitarios de la ciudad de Manizales y Medellín, el 23.1\% ( $n=327)$ obtuvieron puntuaciones que indicaban riesgo de suicidio, de los cuales el $21.3 \%$ ( $n=95)$ fueron hombres y el $24 \%(n=232)$ mujeres. 
Al realizar un análisis de los ítems de la escala, se identificó que el $32.9 \%$ ( $n=465)$ de los estudiantes habían pensado alguna vez en suicidarse, el 19.3\% ( $n=73$ ) le contó a alguien que pensaba en el suicidio, y el $12.5 \%$ ( $n=177)$ habían intentado alguna vez quitarse la vida. E $14.4 \%(n=204)$ de los estudiantes reportaron miembros de la familia que habían intentado suicidarse alguna vez.

Mediante la prueba $U$ de Mann-Whitney se realizó un análisis comparativo entre los grupos con riesgo suicida y los que no presentaron riesgo en función de las variables de inteligencia emocional y autoestima (Tabla 1). Se encontró que los estudiantes con factor de riesgo suicida presentaron, de manera significativa $(p<.01)$, puntuaciones más altas en atención emocional $\left(Z=-2.13 ; p=.034 ; \eta^{2}=.003\right)$ y en autodesprecio $\left(Z=-17.131 ; p<.001 ; \eta^{2}=.21\right)$, siendo esta última variable la que presentó un tamaño del efecto grande $\left(\eta^{2}>\right.$.039) (Fritz et al., 2012). Por el contrario, los estudiantes sin riesgo suicida obtuvieron puntaciones significativamente más altas $(p<.001)$, con un tamaño del efecto entre intermedio a grande $\left(\eta^{2}>.039\right)$ en inteligencia emocional general $\left(Z=-9.37 ; p<.001 ; \eta^{2}=.62\right)$, claridad emocional $\left(Z=-11.25 ; p<0.001 ; \eta^{2}=.09\right)$, regulación emocional $\left(Z=-11.67 ; p<.001 ; \eta^{2}=.096\right)$, autoestima general unifactorial $(Z=-18.342 ; p$ $\left.<.001 ; \eta^{2}=.24\right)$ y autoconfianza $\left(Z=-15.419 ; p<.001 ; \eta^{2}=.17\right)$.

Tabla 1: Diferencias entre estudiantes en función del riesgo suicida y las variables de inteligencia emocional y autoestima

\begin{tabular}{lllllllllllll}
\hline Variables & \multicolumn{3}{l}{ Riesgo suicida } & \multicolumn{4}{l}{ Sin riesgo suicida } & & Z & P & $\boldsymbol{\eta 2}$ \\
\cline { 2 - 12 } & $\mathbf{M}$ & $\mathbf{D E}$ & $\mathbf{R p}$ & $\mathbf{M e}$ & $\mathbf{M}$ & $\mathbf{D E}$ & $\mathbf{R p}$ & $\mathbf{M e}$ & & & \\
Inteligencia emocional & 73.92 & 16.69 & 522.01 & 73 & 83.85 & 15.15 & 763.3 & 84 & -9.37 & .000 & .062 \\
Atención emocional & 27.17 & 7.73 & 749.54 & 28 & 26.37 & 6.91 & 694.85 & 26 & -2.13 & .034 & .003 \\
Claridad emocional & 22.07 & 7.04 & 484.87 & 21 & 27.23 & 6.83 & 774.47 & 27 & -11.25 & .000 & .09 \\
Regulación emocional & 24.69 & 7.52 & 476.68 & 24 & 30.25 & 6.07 & 776.94 & 31 & -11.67 & .000 & .096 \\
Autoestima global & 26.46 & 5.12 & 344.98 & 26 & 33.03 & 4.40 & 816.56 & 34 & -18.34 & .000 & .24 \\
Autoconfianza & 14.97 & 3.03 & 405.67 & 15 & 17.78 & 2.07 & 798.30 & 18 & -15.42 & .000 & .17 \\
Autodesprecio & 13.45 & 2.92 & 1045.39 & 14 & 9.71 & 3.00 & 605.85 & 10 & -17.13 & .000 & 0.21 \\
\hline
\end{tabular}

En la tabla 2 se muestran los resultados del análisis correlacional de las diversas variables de estudio mediante el coeficiente Rho de Spearman. Se encontraron correlaciones estadísticamente significativas $(p<.001)$ de signo positivo entre riesgo suicida, atención emocional y autodesprecio, y correlaciones de signo negativo $(p<.001)$ entre riesgo suicida, inteligencia emocional, claridad emocional, regulación emocional, autoestima y autoconfianza. Estos hallazgos indican que la inteligencia emocional y sus componentes de claridad y regulación, la autoestima global y la autoconfianza son factores protectores del riesgo suicida, y por contrario, la atención emocional y el autodesprecio son factores de riesgo para el suicidio. 
Tabla 2: Coeficiente de correlación de Spearman (Rho) entre el riesgo de suicidio (SRS) y las variables de inteligencia emocional, atención, claridad, regulación, autoestima, autoconfianza y autodesprecio .

\begin{tabular}{|c|c|c|c|c|c|c|c|c|}
\hline Correlaciones & 1 & 2 & 3 & 4 & 5 & 6 & 7 & 8 \\
\hline 1.Riesgo suicida & & $-.291^{* *}$ & $.092^{* *}$ & $-.368^{* *}$ & $-.381^{* *}$ & $-.612^{* *}$ & $-.485^{\star *}$ & $.581^{* *}$ \\
\hline 2.Inteligencia emocional & & & $650^{* *}$ & $.834^{* *}$ & $.763^{* *}$ & $.393^{* *}$ & $.445^{* *}$ & $-.288^{* *}$ \\
\hline 3.Atención emocional & & & & $.303^{* *}$ & $.186^{* *}$ & -.015 & $.065^{*}$ & $.068^{*}$ \\
\hline 4. Claridad emocional & & & & & $.591^{* *}$ & $.439^{* *}$ & $.455^{* *}$ & $-.351^{* *}$ \\
\hline 5.Regulación emocional & & & & & & $.481^{* *}$ & $.502^{* *}$ & $-.385^{* *}$ \\
\hline 6.Autoestima global & & & & & & & $.824^{\star \star}$ & $-.918^{* *}$ \\
\hline 7.Autoconfianza & & & & & & & & $-.566^{* *}$ \\
\hline 8.Autodesprecio & & & & & & & & \\
\hline
\end{tabular}

En la tabla 3 se presentan dos modelos de regresión logística binaria mediante el método de introducción, con el fin de identificar el efecto que tienen las variables de inteligencia emocional y autoestima sobre el factor de riesgo suicida. En el primer modelo se utilizaron las puntuaciones totales de inteligencia emocional y autoestima como variables independientes. En el segundo modelo se utilizaron las dimensiones de atención, claridad y regulación emocional, autoconfianza y autodesprecio como variables independientes. En los dos modelos propuestos el factor de riesgo suicida fue la variable dependiente.

Tanto el primer modelo (chi Hosmer y Lemeshow $15.439 ; g l=8 ; p=.051$ ) como el segundo (chi Hosmer y Lemeshow $=2.622 ; \mathrm{gl}=8 ; \mathrm{p}=.956$ ) presentaron buenos indicadores de bondad de ajuste. El primer modelo evidenció que las variables independientes explicaron entre el $25 \%$ $\left(R^{2}\right.$ Cox y Snell $\left.=.250\right)$ y el $38 \%\left(R^{2}\right.$ Nagelkerke $\left.=.378\right)$ el factor de riesgo suicida, y, el segundo modelo, explicó entre el $26 \%\left(R^{2}\right.$ Cox y Snell $\left.=.264\right)$ y el $40 \%\left(R^{2}\right.$ Nagelkerke $\left.=.40\right)$ la variación del factor de riesgo suicida.

En estos modelos los odds ratios (OR) muestran qué tanto incrementa o disminuye la probabilidad del factor de riesgo suicida según el efecto de las variables independientes. El valor positivo del coeficiente beta $(\beta)$ en las variables independientes indican que son factores que aumentan la probabilidad del riesgo suicida, mientras que, un valor negativo, indican que son variables que reducen la probabilidad del riesgo suicida.

En el modelo 1, se encontró que la inteligencia emocional (OR= .989 IC 95\%=.979-.999) y la autoestima (OR=.768 IC 95\%=.741-.795) disminuyen la probabilidad de riesgo suicida. En el modelo 2 , se encontró que la atención emocional aumentó en un 1\% (OR=1.037 IC 95\%= $1.014-$ $1.060)$ y el autodesprecio en un $1.3 \%$ (OR=1.316 IC 95\%=1.241-1.396) la probabilidad de riesgo 
suicida. Por el contrario, las variables de claridad (OR=.953 IC 95\%=.928-.979) y regulación emocional (OR=.967 IC 95\%=.941-.994) disminuyen en el $1 \%$ y la autoconfianza en un $.8 \%$ (OR=.827 IC 95\%=.771-.888) la probabilidad del riesgo suicida.

Tabla 3: Análisis de regresión logística binaria mediante el método de introducción

\begin{tabular}{|c|c|c|c|c|c|c|c|c|}
\hline \multirow{2}{*}{$\begin{array}{l}\text { Varlables } \\
\text { independientes }\end{array}$} & \multirow[t]{2}{*}{$\beta$} & \multirow[t]{2}{*}{$S E$} & \multirow[t]{2}{*}{$x^{2 W a l d}$} & \multirow[t]{2}{*}{$g l$} & \multirow[t]{2}{*}{$p$} & \multirow[t]{2}{*}{$O R$} & \multicolumn{2}{|c|}{ IC $95 \%$ OR } \\
\hline & & & & & & & Inferior & Superior \\
\hline \multicolumn{9}{|l|}{ Modelo 1.} \\
\hline $\begin{array}{l}\text { Inteligencia } \\
\text { emocional }\end{array}$ & -.011 & .005 & 4,879 & 1 & .027 & 0.989 & .979 & .999 \\
\hline Autoestima & -.264 & .018 & 221,058 & 1 & .000 & 0.768 & .741 & .795 \\
\hline \multicolumn{9}{|l|}{ Modelo 2.} \\
\hline Atención emocional & .036 & .011 & 10,147 & 1 & .001 & 1.037 & 1.014 & 1.060 \\
\hline Claridad emocional & -.048 & .014 & 12,280 & 1 & .000 & 0.953 & .928 & .979 \\
\hline regulación emocional & -.033 & .014 & 5,535 & 1 & .019 & 0.967 & .941 & .994 \\
\hline Autoconfianza & -.189 & .036 & 27,837 & 1 & .000 & 0.827 & .771 & .888 \\
\hline Autodesprecio & .275 & .030 & 83,784 & 1 & .000 & 1.316 & 1.241 & 1.396 \\
\hline
\end{tabular}

Con el objetivo de identificar los efectos directos e indirectos que tienen las variables independientes sobre el riesgo suicida, se estimó un modelo de ecuaciones estructurales a través del método de mínimos cuadrados ponderados, ya que éste permite trabajar con variables que no cumplen el supuesto de normalidad univariante (Byrne, 2016). La tabla 4 muestra los indicadores de bondad de ajuste del modelo propuesto, cuyos resultados muestran que presentan un ajuste adecuado a los datos (McArdle \& Nesselroade, 2014).

Tabla 4: Estadísticas de bondad de ajuste del modelo estructural de predicción del riesgo suicida

\begin{tabular}{lllllllllll}
\hline Modelo & $\chi^{2}$ & $\boldsymbol{g l}$ & $\boldsymbol{p}$ & IF & CFI & NFI & TLI & GFI & AGF & RMSEA \\
\hline $\mathbf{1}$ & 5.572 & 1 & .18 & .994 & .994 & .993 & .909 & .999 & .982 & .057 \\
\hline
\end{tabular}

En el modelo se tomó la variable de atención emocional como mediadora entre la autoconfianza, la claridad emocional y el riesgo suicida. Así mismo, el autodesprecio se ubicó como mediadora entre la autoconfianza, la claridad emocional, la regulación emocional y el riesgo suicida.

Se encontró que la autoconfianza, con un efecto directo negativo, la claridad emocional, con un efecto directo positivo, explicaron en un 10\% la atención emocional $\left(R^{2}=.099\right.$; IC 95\% $=.068$-137; $p<.001$ ). Las dimensiones de atención, claridad y regulación emocional, junto con la autoconfianza explican el $38 \%$ del autodesprecio $\left(R^{2}=.375 ;\right.$ IC $\left.95 \%=.333-.420 ; p<.001\right)$. Todos los efectos directos e indirectos fueron negativos. Finalmente, el riesgo suicida es explicado en un $42 \%\left(R^{2}=.424\right.$; IC $\left.95 \%=.382-.468 ; p<.001\right)$ por el efecto de todas las varia- 
bles independientes. La autoconfianza, la claridad y regulación emocional aportaron efectos directos negativos y la atención emocional y el autodesprecio aportaron efectos directos positivos.

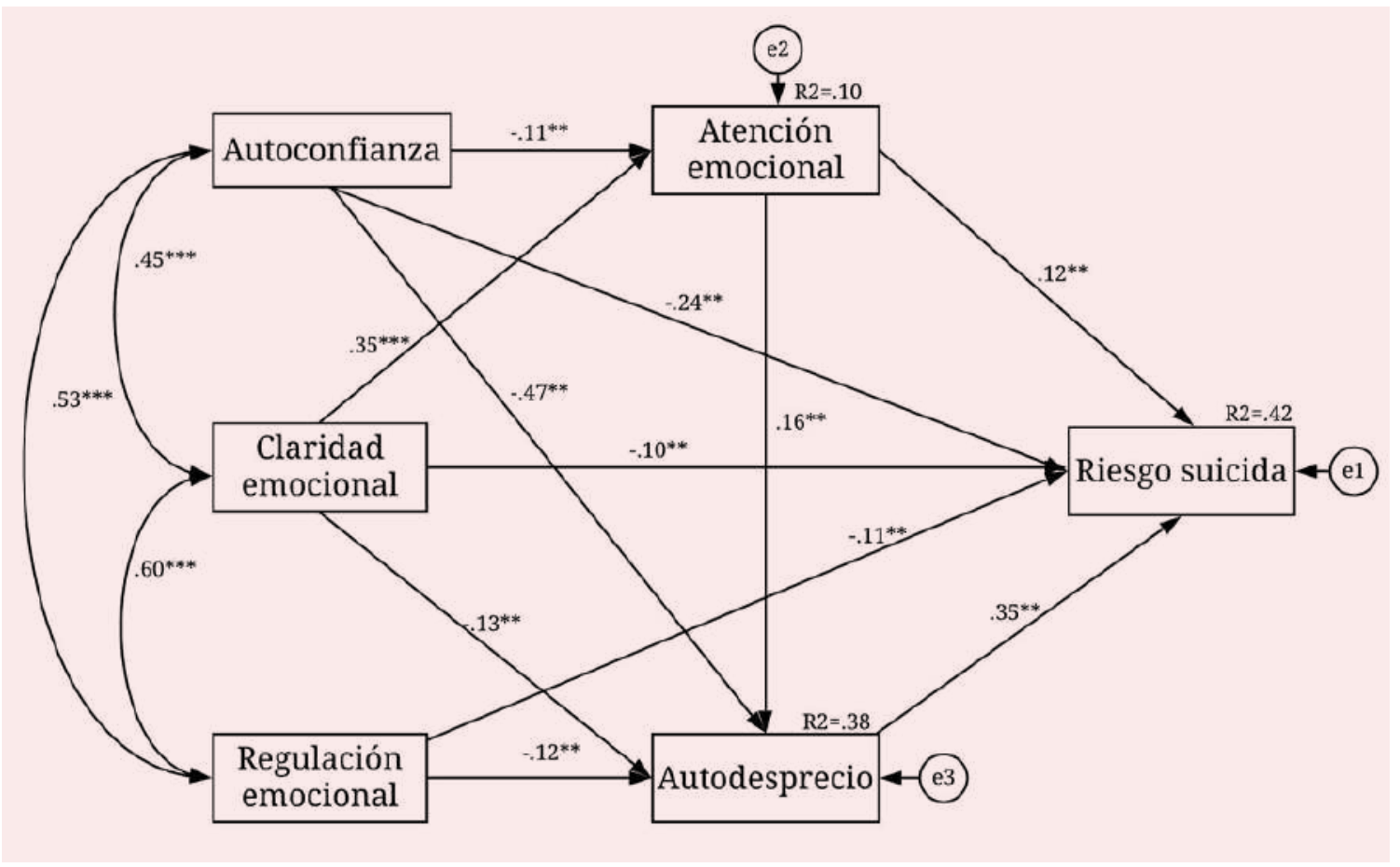

Figura 1: Modelo de ecuaciones estructurales del riesgo suicida.

La tabla 5 muestra los efectos estandarizados totales, directos e indirectos de las variables de estudio. Las variables de autoconfianza y regulación emocional aportaron efectos totales, directos e indirectos significativos $(p<.001$ ) y negativos sobre el riesgo suicida. La claridad emocional presentó un efecto directo negativo sobre el riesgo suicida. La atención emocional y el autodesprecio aportaron efectos totales, directos e indirectos significativos ( $p<.001)$ y positivos sobre el riesgo suicida. Todos los efectos fueron estadísticamente significativos ( $p<$ $.01)$. 
Tabla 5: Efectos totales, directos e indirectos estandarizado de las variables predictoras sobre las variables de respuesta.

\begin{tabular}{|c|c|c|c|c|c|c|c|c|c|c|}
\hline & & \multicolumn{3}{|c|}{ Atención emocional } & \multicolumn{3}{|c|}{ Autodesprecio } & \multicolumn{3}{|c|}{ Riesgo suicida } \\
\hline & & \multirow[t]{2}{*}{ Efectos } & \multicolumn{2}{|l|}{$1 C 95 \%$} & \multirow[t]{2}{*}{ Efectos } & \multicolumn{2}{|l|}{$1 C 95 \%$} & \multirow[t]{2}{*}{ Efectos } & \multicolumn{2}{|l|}{$1 C 95 \%$} \\
\hline & & & Inferior & superior & & Inferior & superior & & Inferior & superior \\
\hline \multirow[t]{3}{*}{ Autoconfianza } & Total & $-.108^{* *}$ & -.169 & -.047 & $-.485^{* \prime}$ & -.533 & -.433 & $-.422^{\prime \prime}$ & -.478 & -.366 \\
\hline & Directo & $-.108^{* *}$ & -.169 & -.047 & $-.468^{\circ *}$ & -.516 & -.417 & $-.241^{\prime \prime}$ & -.303 & -.178 \\
\hline & Indirecto & -- & -- & -- & $-.017^{*}$ & -.029 & -.007 & $-.181^{* *}$ & -.214 & -.15 \\
\hline \multirow{3}{*}{$\begin{array}{l}\text { Regulación } \\
\text { emocional }\end{array}$} & Total & -- & -- & -- & $-.115^{* \prime}$ & -.172 & -.061 & $-.147^{\prime \prime}$ & -.204 & -.089 \\
\hline & Directo & -- & -- & -- & $-.115^{* *}$ & -.172 & -.061 & $-.107^{* *}$ & -.161 & -.052 \\
\hline & Indirecto & -- & - & -- & -- & -- & -- & $-.04^{* *}$ & -.062 & -.021 \\
\hline \multirow{3}{*}{$\begin{array}{l}\text { Claridad } \\
\text { emocional }\end{array}$} & Total & $.348^{*}$ & .287 & .409 & $-.069^{*}$ & -.122 & -.013 & $-.086^{\circ}$ & -.142 & -.029 \\
\hline & Directo & $348^{\circ *}$ & .287 & .409 & $-.125^{* \prime}$ & -.179 & -.07 & $-.104^{* *}$ & -.157 & -.05 \\
\hline & Indirecto & -- & -- & -- & $.056^{\prime \prime}$ & .038 & .075 & .018 & -.011 & .046 \\
\hline \multirow{3}{*}{$\begin{array}{l}\text { Atención } \\
\text { emocional }\end{array}$} & Total & -- & -- & -- & $.161^{*}$ & .113 & .207 & $.176^{* *}$ & .129 & .22 \\
\hline & Directo & -- & -- & -- & $.161^{* \prime}$ & .113 & .207 & $.12^{\prime \prime}$ & .076 & .164 \\
\hline & Indirecto & -- & -- & -- & -- & -- & -- & $.056^{* \prime}$ & .039 & .074 \\
\hline \multirow[t]{3}{*}{ Autodesprecio } & Total & --- & --- & -- & -- & -- & -- & $.347^{* *}$ & .295 & .401 \\
\hline & Directo & -- & -- & -- & -- & -- & -- & $.347^{* \prime}$ & .295 & .401 \\
\hline & Indirecto & -- & -- & -- & -- & -- & -- & --- & - & -- \\
\hline
\end{tabular}

\section{Discusión}

El presente estudio expone la relación del riesgo suicida con las variables inteligencia emocional y autoestima en estudiantes universitarios de dos ciudades colombianas.

En la población estudiantil de ambas ciudades se encuentra una prevalencia del riesgo suicida del $23.1 \%$, siendo el porcentaje mayor en mujeres (24\%) que en hombres $(21.3 \%)$. Estos resultados concuerdan con lo encontrado en otros estudios (Chatard et al., 2009).

En general, se encuentra en la población estudiantil que a menor riesgo suicida mayor inteligencia emocional, claridad emocional, regulación emocional, autoestima y autoconfianza. Estos resultados tienen similitudes con otras investigaciones (Carmona et al., 2017; Chatard et al., 2009; Rosselló \& Berríos, 2004); se ha encontrado que la claridad y la regulación emocional tienen una correlación negativa y significativa con la ideación suicida, y a su vez, que la baja autoestima aumenta hasta en 3 veces el riesgo de tener ideación e intentos suicidas (Sarmiento \& Villalobos, 2011).

Considerando que en el estudio el autodesprecio se ubicó como variable mediadora entre la autoconfianza, la claridad emocional, la regulación emocional y el riesgo suicida, se encuentra un contraste con un estudio reciente que muestra como la baja autoconfianza emocional predispondría a pensamientos suicidas utilizando un modelo de análisis de regresión que mostró que el modelo de mejor ajuste era un modelo de efectos directos (acumulativo) en el que la emocionalidad y la emoción negativas y la autoconfianza contribuyó a la ideación suicida (Deeley \& Love, 2013) y ésta a su vez es un predictor del riesgo suicida (Gómez et al., 2019). 
Se ha reportado que la tendencia suicida implica discrepancias intolerables, actuaciones reales y estándares ideales que conllevan la experimentación de sensación de fracaso de acuerdo con las normas propias, autorrestricción emocional en las relaciones, fuertes sentimientos de soledad y rechazo de sí mismo (Rasmussen et al., 2015), algunos de ellos asociados a trastornos psicológicos, aunque se ha mostrado cómo la baja autoestima se correlaciona con la intencionalidad suicida, independientemente de la gravedad de la depresión (Perrot et al., 2018). Pero en pacientes suicidas se ha mostrado sin embargo que, dentro de las perspectivas de tratamiento con la presencia de trastornos, la gravedad del mismo si se asocia con la opción o no de recuperación de la salud emocional (SantamarinaPerez et al., 2020), lo cual hace pensar en la utilidad de los predictores analizados en estudios respecto a la expectativa positiva de las intervenciones.

En el análisis de resultados del presente estudio se identifica que aquellos estudiantes con factor de riesgo suicida presentan una mayor puntuación en atención emocional y en autodesprecio. Este hallazgo es semejante a lo encontrado por Suárez y Caballero (2016), al hallar una correlación negativa significativa entre claridad y regulación emocional e ideación suicida, mas no encontrar relación con las ideas suicidas y la habilidad para atender a las emociones; sin embargo, se ha mostrado que la desregulación emocional es un predictor de la ideación suicida (Neacsiu et al., 2018).

Ahora, desde el punto de vista de la regulación emocional, se ha mostrado equivalencia de resultados en jóvenes con y sin intentos de suicidio, mostrando representaciones desorganizadas, especialmente en la afectividad (Pallini et al., 2020), lo que implica no subestimar el valor predictivo que tiene la ideación respecto del riesgo suicida asociado a la inteligencia emocional. Mediante análisis de regresión se han encontrado asociaciones significativas para regulación emocional, específicamente falta de claridad emocional antes del primer episodio suicida, y aunque se reconocen las dificultades en la evaluación temprana y comprensión de las emociones y su modulación en el aumento del riesgo suicida, brindan buenas herramientas para una información temprana de tipo preventiva (Palmer et al., 2019).

En un estudio en que se utiliza como instrumento de medición de la inteligencia emocional el WLEIS (Extremera et al., 2018), se encuentra similar al presente, que la inteligencia emocional está correlacionada negativamente con el riesgo suicida. Por otro lado, en una investigación de corte cualitativo (Rasmussen et al., 2015), en la que se explora la relación entre la autoestima y suicidio se encuentra que, en general, los jóvenes que cometieron suicidio no contaban con la capacidad para apreciar su valor personal y necesitaban de la aprobación constante de personas significativas para ellos; su autoestima dependía de sus éxitos y apariencia física, la crisis suicida sucedía debido a la intensidad emocional incontrolable que surgía al no cumplir con los propios estándares de perfección. 
Estos hallazgos revelan que, independientemente del instrumento de medición de la inteligencia emocional y del carácter cualitativo o cuantitativo de las investigaciones, se encuentra relación significativa entre la conducta suicida y la inteligencia emocional como factor predictivo y como posible recurso psicosocial a fortalecer en las personas (DomínguezGarcía \& Fernández-Berrocal, 2018).

Los hallazgos mencionados son coherentes con lo encontrado en diversas investigaciones cuantitativas a nivel nacional (Siabato \& Salamanca, 2015; Ceballos et al., 2015) e internacional (Abdollahi et al., 2016; Gómez et al., 2018; Sánchez et al., 2014; Bruns \& Letcher, 2018), al considerar que tanto la autoestima, la valía persona y la autoconfianza, así como la capacidad para reconocer, comprender, regular y expresar adecuadamente las emociones, especialmente la rabia, la tristeza, la culpa y la ansiedad, son factores de protección ante el riesgo suicida y, además, son predictores de la salud mental (Schutte et al., 2007) en contraste con el efecto negativo que implica una menor inteligencia emocional y una mayor tendencia de riesgo suicida (Gómez et al., 2018).

A este respecto, Oquendo (2010) plantea que la comprensión de la conducta suicida está relacionada con la interacción entre factores de diátesis y estrés y, de este modo, a menor vulnerabilidad psicosocial mayor es la capacidad de la persona para afrontar las situaciones estresantes en la vida diaria. La inteligencia emocional y la autoestima son factores que al funcionar adecuadamente posibilitan afrontar de manera adecuada el estrés psicosocial y disminuir el factor de riesgo suicida (Kuhlberg et al., 2010; Mamani et al., 2018), especialmente asociado al peso de la vivencia afectiva.

Los aspectos emocionales, afectivos y de pareja se relacionan como los factores de mayor impacto en el equilibrio de la salud mental de los universitarios (Núñez, 2004), por cuanto ello implica, no solo el ajuste contextual relacional (Quintana-Orts et al., 2019), sino también los vínculos afectivos asociados a las relaciones familiares, la autoeficacia y autoestima (Pereira et al., 2018).

Es muy interesante la relación del suicidio con aspectos psicológicos profundos, ya que se ha mostrado que el dolor mental insoportable y la depresión, pero no la desesperanza, podrían predecir cambios en estado de intento de suicidio, considerando la depresión y la desesperanza evaluadas simultáneamente, pero solo el dolor mental insoportable tiene un poder predictivo único y directo en el riesgo suicida (Lambert et al., 2020).

Tanto la inteligencia emocional como la autoestima son recursos personales que los sujetos desarrollan a lo largo de su vida y les permiten afrontar los distintos eventos vitales de una manera que enriquezca su personalidad y su salud mental al mejorar su calidad de vida (Faure 
et al., 2018); de ahí la importancia de asumirlos como recursos psicosociales que se deben fortalecer en los adolescentes y en los jóvenes como medida preventiva ante el riesgo de conducta suicida (Aradilla-Herrero et al., 2014).

Estos hallazgos ayudan a la explicación epidemiológica de la conducta suicida en tanto que la inteligencia emocional y la autoestima son factores que explican los cambios en la prevalencia de la ideación, intento y suicidio consumado en poblaciones homologables al presente estudio (Cha \& Nock, 2009).

El presente estudio incluso podría orientar la tendencia de estudios actuales al impactar variables relacionadas como la gratitud y el optimismo (Sánchez-Álvarez et al., 2020), estado de ánimo negativo crónico y las fortalezas emocionales como amortiguadores específicos para el abordaje de problemáticas de suicidio (Chang et al., 2021) y aspectos relacionados con soledad y suicidio (Chang et al., 2019).

Finalmente, si bien los resultados del presente estudio no se orientaron a establecer la relación de las variables respecto al sexo, valdría la pena para futuros estudios detallar su análisis, pues se tiene evidencia no concluyente respecto a que los hombres presentan puntuaciones más altas en inteligencia emocional y en autoconfianza (Mérida-López et al., 2018). Este hallazgo está relacionado con que los hombres tienen una mayor autoaceptación, tanto de sus aspectos positivos como negativos en relación con las mujeres (Chatard, et al., 2009; Domínguez, et al., 2015; González-Fuentes \& Andrade, 2013), y además contrasta con la tasa de mortalidad por suicidio entre jóvenes de 20 a 24 reportada en Colombia en 2018 por Medicina Legal, con una cifra global de suicidas en hombres tres veces mayor que en mujeres.

Así mismo, se podrían requerir nuevos estudios que contrasten las variables aquí estudiadas con la edad, ya que se tienen evidencias de que ésta es un factor importante en relación con el riesgo suicida, la autoestima y la capacidad para regular las emociones al evidenciar promedios más altos en inteligencia emocional y autoestima respecto a muestras entre 26 a 30 años, y muestras entre 16 a 20 y de 21 a 25 años, lo cual prevé que a mayor edad menor es el riesgo suicida (Crandall et al., 2018).

Los datos arrojados por este estudio, sin duda ubican como centro de importancia los sistemas de atención por parte de las instituciones universitarias, lo cual implica que los sistemas de trabajo en Bienestar Universitario tengan un abordaje de esta problemática asociando los factores contextuales múltiples e individuales, aspecto que se relaciona con datos recientes que muestran que los estudiantes universitarios tienen más probabilidades de 
considerar o intentar suicidarse, lo que subraya la necesidad de intervenciones enfocadas en la prevención del riesgo de suicidio con esta población (Fox et al., 2020). Ello se relaciona con el uso de modelos de trabajo menos restringidos respecto de la atención integral en poblaciones universitarias, lo que requiere tener un nivel funcional más sistémico y articulado, tal como ya lo han sugerido Núñez et al. (2006).

\section{Reconocimientos}

Este artículo se deriva del proyecto de investigación "Factores de riesgo y predictores psicosociales y neuropsicológicos asociados a la conducta suicida en estudiantes universitarios entre 16 a 25 años", liderado y financiado por la Universidad Católica Luis Amigó, y en colaboración con la Universidad de Medellín y la Asociación Psicológica lberoamericana de Clínica y Salud-APICSA.

\section{References}

Abdollahi, A., Carlbring, P., Khanbani, M., \& Abdollahi, S. (2016). Emotional intelligence moderates perceived stress and suicidal ideation among depressed adolescent inpatients. Personality and Individual Differences, 102, 223-228. https://doi.org/10.1016/ j.paid.2016.07.015

Antón-San-Martín, J. M., Sánchez-Guerrero, E., Pérez-Costilla, L., Labajos-Manzanares, M.-T., de-Diego-Otero, Y., Benítez-Parejo, N., Comino-Ballesteros, R., Perea-Milla, E., RamosMedina, V., \& López-Calvo, A. (2013). Factores de riesgo y protectores en el suicidio. Un estudio de casos y controles mediante la autopsia psicológica. Anales de Psicología, 29(3), 810-815. https://doi.org/10.6018/analesps.29.3.175701

Aradilla-Herrero, A., Tomás-Sábado, J., \& Gómez-Benito, J. (2014). Associations between emotional intelligence, depression and suicide risk in nursing students. Nurse Education Today, 34(4), 520-525. https://doi.org/10.1016/j.nedt.2013.07.001

Atienza, F., Moreno, Y., \& Balaguer, I. (2000) Análisis de la Dimensionalidad de la Escala de Autoestima de Rosenberg en una Muestra de Adolescentes Valencianos. Revista de Psicología. Universitas Tarraconensis, 22, 29-42. https://tinyurl.com/yygl73nh

Aydin I, Algin A, Poyraz, M. K., \& Kalenderoglu A. (2018). An analysis with Rotter Internal- External Locus of Control Scale, Rosenberg Self-Esteem Scale, and the Barratt Impulsivity Scale on patients who attempted to recurrent suicide. Eurasian Journal Ofemergency Medicine, 17(3): 109-12. https://doi.org/10.5152/eajem.2018.39306

Blandón, O. M., Carmona, J. A., Mendoza, M., \& Medina, O. (2015). Ideación suicida y factores asociados en jóvenes universitarios de la ciudad de Medellín. Revista Archivo Médico de Camagüey, 19(5), 469-478. https://tinyurl.com/yxuwamqw 
Bruns, K., \& Letcher, A. (2018). Protective Factors as Predictors of Suicide Risk Among Graduate Students. Journal of College Counseling, 7(21), 111-124. https://doi.org/10.1002/ jocc. 12091

Byrne, B. (2016). Structural equation modeling with Amos (3rd ed.). New York, NY: Routledge.

Cañón, S. C., Castaño, J. J., Atehortúa, B. E., Botero, P., García, L. K., Rodríguez, L. M., Tovar, C. A., \& Rincón, E. (2012). Factor de riesgo para suicidio según dos cuestionarios y factores asociados en población estudiantil de la Universidad de Manizales (Colombia), 2011. Psicología desde el Caribe, 29 3), 632-664. https://tinyurl.com/y4uedfgs

Carmona, J., Carmona, D., Maldonado, N., Rivera, C., Fernández, O., Cañón, S., Alvarado, S., Jaramillo, J., Narváez, M., Fandiño, D., Vélez, D., \& Velázquez, H. (2017). El suicidio y otros comportamientos autodestructivos en jóvenes universitarios de Colombia y Puerto Rico: acciones, interacciones y significaciones. Manizales: Universidad de Manizales. https://tinyurl.com/y346vv3a

Ceballos-Ospino,c G., Suarez-Colorado, Y., Suescún- Arregocés, J., Gamarra-Vega, L., González, K., \& Sotelo-Manjarres, A. (2015). Ideación suicida, depresión y autoestima en adolescentes escolares de Santa Marta. Duazary: Revista Internacional de Ciencias de la Salud, 12(1), 15 - 22. https://doi.org/10.21676/2389783X.1394

Cha, C. B., \& Nock, M. K. (2009). Emotional intelligence is a protective factor for suicidal behavior. Journal of the American Academy of Child \& Adolescent Psychiatry, 48(4), 422430. https://doi.org/10.1097/CHI.0b013e3181984f44

Chang, E., Martos, T., Sallay, V., Chang, O., Wright, K., Najarian, A., \& Lee, J. (2017) Examining optimism and hope as protective factors of suicide risk in hungarian college students: is risk highest among those lacking positive psychological protection?. Cognitive Therapy and Research, 41(2), 278-288. https://doi.org/10.1007/s10608-016-9810-0

Chang, E. C., Chang, O. D., Lucas, A. G., Li, M., Beavan, C. B., Eisner, R. S., McManamon, B. N., Rodriguez, N. S, Katamanin, O. M, Bourke, E. C, De la Fuente, A., Cardeñoso, O., Wu K., Yu, E. A., Jeglic, E. L., \& Hirsch, J .K. (2019). Depression, loneliness, and suicide risk among Latino college students: a test of a psychosocial interaction model. Social Work, 64(1), 5160. https://doi.org/10.1093/sw/swy052

Chang, E. C., Sánchez-Álvarez, N., Rey, L., Extremera, N., \& Chang, O. D. (2021). Chronic negative mood and emotional strengths: Some evidence for using emotions as a specific buffer to the problem of suicide. Personality and Individual Differences, 168. https://doi.org/10.1016/j.paid.2020.110384 
Chatard, A., Selimbegović, L., \& Konan, P. (2009). Self-esteem and Suicide Rates in 55 Nations. European Journal of Personality, 23(1), 19-32. https://doi.org/10.1002/per.701

Cogollo, Z., Campo-Arias, A., \& Herazo, E. (2015). Escala de Rosenberg para autoestima consistencia interna y dimensionalidad en estudiantes de Cartagena, Colombia. Psychologia: Avances en la Disciplina, 9(2), 61-71. https://tinyurl.com/y2asu765

Crandall, A., Allsop, Y., \& Hanson, C. (2018). The longitudinal association between cognitive control capacities, suicidality, and depression during late adolescence and Young adulthood. Journal of Adolescence, 65, 167-176. https://doi.org/10.1016/j.adolescence. 2018.03.009

Deeley, S. T., \& Love, A. W. (2013). A Preliminary Investigation into the Emotion Self-Confidence Model of Suicidal Ideation in Adolescents. Archives of Suicide Research, 17(2), $161-172$. https://doi.org/10.1080/13811118.2013.776458

Domínguez-García, E., \& Fernández-Berrocal, P (2018). The association between emotional intelligence and suicidal behavior: a systematic review. Frontiers in Psychology, 9. https://doi.org/10.3389/fpsyg.2018.02380

Domínguez, C. C., Colorado, Y. P. S., \& Carbonó, H. D. B. (2015). Características de inteligencia emocional en un grupo de universitarios con y sin ideación suicida. Revista CES Psicología, 8(2), 138-155. https://tinyurl.com/y5k6svff

Extremera N., Quintana-Orts C., Mérida-López S., \& Rey, L. (2018) Cyberbullying Victimization, Self-Esteem and Suicidal Ideation in Adolescence: Does Emotional Intelligence Play a Buffering Role? Frontiers in Psychology. 9(367) 1-9. https://doi.org/10.3389/fpsyg. 2018.00367

Faure, M., Urquidi, C., Bustamante, F., Florenzano, R., Ampuero, K., Terán, L., Figueroa, M., Farí -as, M., Rueda, M.L., \& Giacaman, E. (2018). Association of health-related quality of life and suicidal risk in adolescents: A cross-sectional study. Revista chilena de pediatría, 89(3), 318-324. https://doi.org/10.4067/S0370-41062018005000103

Fernández, P., \& Extremera, N. (2005). La Inteligencia Emocional y la educación de las emociones desde el Modelo de Mayer y Salovey. Revista Interuniversitaria de Formación del Profesorado, 19(3), 63-93. https://tinyurl.com/y4pmukc7

Fernández-Berrocal, P., Extremera, N., \& Ramos, N. (2004). Validity and reliability of the Spanish version of the Trait Meta-mood Scale. Psychological Reports, 94, 751-755. https://doi.org/10.2466/pr0.94.3.751-755

Forrester, R. L., Slater, H., Jomar, K., Mitzman, S., \& Taylor, P. J. (2017). Self-esteem and nonsuicidal self-injury in adulthood: A systematic review. Journal of Affective Disorders, 221, 172-183. https://doi.org/10.1016/j.jad.2017.06.027 
Fox, M. L., James, T. G., \& Barnett, S. L. (2020). Suicidal Behaviors and Help-Seeking Attitudes Among Deaf and Hard-of-Hearing College Students. Suicide Life Threat Behav, 50, 387396. https://doi.org/10.1111/sltb.12595

Fritz, C. O., Morris, P. E., \& Richler, J. J. (2012). Effect size estimates: Current use, calculations, and interpretation. Journal of Experimental Psychology: General, 141, 2-18. https://doi.org/10.1037/a0024338

Gardner, H. (1983). Frames of mind: The theory of multiple intelligences. New York, NY: Basic Books.

Gerbing, D. W., \& Anderson, J. C. (1988). An Updated Paradigm for Scale Development Incorporating Unidimensionality and Its Assessment. Journal of Marketing Research, 25(2), 186192. https://doi.org/10.2307/3172650

Gómez, M. J., Limonero, J. T., Toro, J., Montes, J., \& Tomas, J. (2018). Relación entre inteligencia emocional, afecto negativo y riesgo suicida en jóvenes universitarios. Estrés y Ansiedad, 24, 18-23. https://doi.org/10.1016/j.anyes.2017.10.007 1134-7937

Gómez, T. A., Núñez, C., Agudelo, O. M., \& Caballo, V. (2019). Predictores psicológicos del riesgo suicida en estudiantes universitarios. Behavioral Psychology, 27(3), 391-413. https://tinyurl.com/y5bkyay8.

Gómez, A. S., Núñez, C., Agudelo, M. P., \& Grisales, A. M. (2020). Riesgo e Ideación Suicida y su Relación con la Impulsividad y la Depresión en Adolescentes Escolares. Revista Iberoamericana de Diagnóstico y Evaluación - e Avaliação Psicológica. RIDEP, 54(1), 147163. https://doi.org/10.21865/RIDEP54.1.12

Gómez-Lugo, M., Espada, J. P., Morales, A., Marchal-Bertrand, L., Soler, F., \& Vallejo Medina, P. (2016). Adaptation, Validation, Reliability and Factorial Equivalence of the Rosenberg Self-Esteem Scale in Colombian and Spanish Population. Spanish Journal of Psychology, 19, e66. https://doi.org/10.1017/sjp.2016.67

González-Fuentes, M., \& Andrade Palos, P. (2013). Auto-aceptación como factor de riesgo para el intento de suicidio en adolescentes. Salud \& Sociedad, 4(1), 26-35. https://doi.org/10.22199/S07187475.2013.0001.00002

Hayes, A. F. (2018). Introduction to mediation, moderation, and conditional process analysis (2nd ed.). New York, NY: The Guilford Press.

Hernández-Sampieri, R., Fernández-Collado, C. \& Baptista-Lucio, P. (2014). Metodología de la investigación (6a ed.). México: Mc Graw Hill. 
Kleiman, E., \& Liu, R. (2013). Social support as a protective factor in suicide: Findings from two nationally representative samples. Journal of Affective Disorders, 150, 540-545. https://doi.org/10.1016/j.jad.2013.01.033

Kuhlberg, J. A., Peña, J. B., \& Zayas, L. H. (2010). Familism, parent-adolescent conflicts, selfesteem, internalizing behaviors and suicide attempts among adolescent Latinas. Child Psychiatry \& Human Development, 41(4), 425-440. https://doi.org/10.1007/s10578-0100179-0

IBM Corporation (2017). IBM SPSS Statistics for Windows, Versions 25.0 [programa de ordenador]. Armonk, NY: IBM Corporation

IBM Corporation (2017). AMOS for Windows, Versions 24.0 [programa de ordenador]. Armonk, NY: IBM Corporation

Instituto Nacional de Medicina Legal y Ciencias Forenses. (2020). Boletines estadísticos. Bogotá, Colombia. https://tinyurl.com/y6a4sztp

Lambert, C. E., Troister, T., Ramadan, Z., Montemarano, V., Fekken, G. C., \& Holden, R. R. (2020). Psychache Predicts Suicide Attempter Status Change in Students Starting University. Suicide Life Threat Behav, 50, 668-678. https://doi.org/10.1111/sltb.12624

Linehan, M., \& Goodstein, J. (1983). Reasons for Staying Alive When You Are Thinking of Killing Yourself: The Reasons for Living Inventory. Journal of Consulting and Clinical Psychology, 51(2), 276-286. https://doi.org/10.1037//0022-006x.51.2.276

Mamani, O., Brousett, M., Ccori, D., \& Villasante, K. (2018). La inteligencia emocional como factor protector en adolescentes con ideación suicida. Duazary, 15(1), 39-50. https://doi.org/10.21676/2389783X.2142

McArdle, J. J., \& Nesselroade, J. R. (2014). Basics of structural equation modeling. In J. J. McArdle \& J. R. Nesselroade. Longitudinal data analysis using structural equation models (pp. 27-37). Washington, DC, US: American Psychological Association

Martínez, C., Gálvez, C., Quiroz, C., Vicencio, B., \& Tomicic, A. (2014) He decidido vivir: Factoes que disuaden a una persona de cometer suicido. Revista Argentina de Clínica Psicológica, 23(3), 267-280. https://tinyurl.com/y3elru2n

Mayer, J. D, \& Salovey, P. (1993). The intelligence of emotional inteligence. Intelligence, 17, 433 -442. https://doi.org/10.1016/0160-2896(93)90010-3

Mayer, L., Rüsch, N., Frey, L.M., Nadorff, M. R., Drapeau, C. W., Sheehan, L., \& Oexle, N. (2020). Anticipated Suicide Stigma, Secrecy, and Suicidality among Suicide Attempt Survivors. Suicide Life Threat Behav, 50, 706-713. https://doi.org/10.1111/sltb.12617

McDonald, R. P. (1999). Test theory: A unified treatment. Mahwah: Lawrence Erlbaum Associates, Inc. 
Neacsiu, A. D., Fang, C. M., Rodriguez, M., \& Rosenthal, M. Z. (2018). Suicidal behavior and problems with emotion regulation. Suicide and Life-Threatening Behavior, 48(1), 52-74. https://doi.org/10.1111/sltb.12335

Núñez, A., Castrillón, D., \& Bañol, J. (2002). Hacia una lectura de vivencias, relatos juveniles y habilidades parar vivir en estudiantes, desde el proyecto para la promoción de la salud y prevención integral de la Universidad de Manizales. En C. Núñez, M. Ramírez, L. Jurado, P. Gíl, \& J. Restrepo (Eds.). Promoción de la salud y jóvenes: experiencias universitarias (pp. 133-150). Manizales: Alcaldía de Manizales, Grupo Interinstitucional de Programas para Adolescentes GIPA, Universidad de Manizales, Universidad de Caldas. https://tinyurl.com/yxknllzh

Núñez, C. (2004). Jóvenes universitarios y salud. Vivir la Universidad (tesis de maestría). Manizales: Universidad de Manizales. https://doi.org/10.13140/RG.2.2.26609.38246

Núñez, C. Tobón, S., Bañol, R., \& Arias, D. (2006). Sistema articulado de bienestar universitario. Revista hacia la promoción de la salud, 11, 61 - 72. https://tinyurl.com/y6dsdr9o

Oquendo, M. (2010). Predictores clínicos de conducta suicida. Abordaje prospectivo (tesis doctoral). Universidad Autónoma de Madrid, España. https://tinyurl.com/y29me3rb

Organización Mundial de la Salud (OMS). (2019). Suicidio. Centro de Prensa. https://tinyurl. com/y68wmwlx

Pallini, S., Terrinoni, A., lannello, S., Cerutti, R., Ferrara, M., Fantini, F., \& Laghi, F. (2020). Attachment-Related Representations and Suicidal Ideations in Nonsuicidal Self-Injury Adolescents with and without Suicide Attempts: A Pilot Study. Suicide Life Threat Behav, 50, 909-920. https://doi.org/10.1111/sltb.12633

Palmer, C., Connor, C., Channa, S., Lavis, A., Leung, N., Parsons, N., y Birchwood, M. (2019), The Development of First-Episode Direct Self-Injurious Behavior and Association with Difficulties in Emotional Regulation in Adolescence. Suicide Life Threat Behav, 49, 12661280. https://doi.org/10.1111/sltb.12512

Patsali, M. E., Mousa, D. V., Papadopoulou, E. V. K., Papadopoulou, K. K. K., Kaparounaki, C. K., Diakogiannis, I., \& Fountoulakis, K. N. (2020). University students' changes in mental health status and determinants of behavior during the COVID-19 lockdown in greece. Psychiatry Research, 292. https://doi.org/10.1016/j.psychres.2020.113298

Pereira, A. S., Willhelm, A. R., Koller, S. H., \& Almeida, R. M. M. D. (2018). Risk and protective factors for suicide attempt in emerging adulthood. Ciencia \& Saude Coletiva, 23(11), 3767-3777. https://doi.org/10.1590/1413-812320182311.29112016 
Perrot, C., Vera, L., \& Gorwood, P. (2018). La faible estime de soi est corrélée à l'intentionnalité suicidaire, indépendamment de la sévérité de la dépression. L'Encéphale, 44(2), 122127. https://doi.org/10.1016/j.encep.2016.10.003

Plutchik, R., \& Van Praag, H. M. (1989). The measurement of suicidality, aggressivity and impulsivity. Progress in Neuro-Psychopharmacology \& Biological Psychiatry, 13, 23-24. https://doi.org/10.1016/0278-5846(89)90107-3

Quintana-Orts, C., Rey, L., Mérida-López, S., \& Extremera, N. (2019). What bridges the gap between emotional intelligence and suicide risk in victims of bullying? A moderated mediation study. Journal of Affective Disorders, 245, 798-805. https://doi.org/10.1016/j.jad.2018.11.030

Quintana-Ortz, S., Merida-López, S., Rey, L., Nieto, F., \& Extermera, N. (2020), Untangling the Emotional Intelligence-Suicidal Ideation Connection: The Role of Cognitive Emotion Regulation Strategies in Adolescents. Journal of clinical medicine, 9, 3116. https://doi.org/10.3390/jcm9103116

Ramírez, D. C., \& Olivella, G. (2014). Validación de la escala de riesgo suicida de Plutchik, en población civil colombiana y policías activos de la policía nacional de Colombia (tesis de maestría). Bogotá: Fundación Universitaria Konrad Lorenz.

Rasmussen, M., Dyregrov, K., Haavind, H., Leenaars, A., \& Dieserud, G. (2015). The Role of SelfEsteem in Suicides Among Young Men. OMEGA-Journal of Death and Dying, 77(3), 217239. https://doi.org/10.1177/0030222815601514

Rengasamy, M., Sylvester, C., Shulman, J., \& Pizon, A. (2020). Contemporary Characteristics and Lethality Correlates of Serious Suicide Attempts in Children and Adolescents. Suicide Life Threat Behav, 50, 714-723. https://doi.org/10.1111/sltb.12615

Rosselló, J., \& Berríos H., M. (2004). Ideación Suicida, Depresión, Actitudes Disfuncionales, Eventos de Vida Estresantes y Autoestima en una Muestra de Adolescentes Puertorriqueños/as. Interamerican Journal of Psychology, 38(2), 295-302. https://tinyurl.com/yytebvsb

Rubio, G., Montero, I., Jáuregui, J., Villanueva, R., Casado, M. A., Marín, J. J., \& Santo-Domingo, J. (1998). Validación de la escala de riesgo suicida de Plutchik en población española. Archivos de Neurobiología, 61(2), 143-52.

Sa, B., Ojeh, N., Majumder, M. A. A., Nunes, P., Williams, S., Rao, S. R., \& Youssef, F. F. (2019). The Relationship Between Self-Esteem, Emotional Intelligence, and Empathy Among Students From Six Health Professional Programs. Teaching and Learning in Medicine, 1-8. https://doi.org.10.1080/10401334.2019.1607741

Salovey, P. \& Grewal, D. (2006). Inteligencia Emocional. Revista Mente y Cerebro, 16, 10-20. https://tinyurl.com/y26hgaar 
Salovey, P., \& Mayer, J. D. (1990). Emotional intelligence. Imagination, Cognition and Personality, 9. 185-211. https://doi.org/10.2190/DUGG-P24E-52WK-6CDG

Salovey, P., Mayer, J. D., Goldman, S. L., Turvey, C., \& Palfai, T. P. (1995). Emotional attention, clarity and repair: Exploring emotional intelligence using the Trait Meta-mood Scale. En J. W. Pennebaker (Ed.), Emotion, disclosure, and health (pp. 125-151). Washington, DC: American Psychological Association

Sánchez, D., Muela, J., \& García, A., (2014). Variables psicológicas asociadas a la ideación suicida en estudiantes. International Journal of Psychology and Psychological Therapy, 14 (2), 277-290. https://tinyurl.com/yxv9d5cz

Sánchez, D., Domínguez, M., Hernández, L., \& González, I. (2015). Estrategias y estilos de afrontamiento en adolescentes con intento suicida. Revista de Ciencias Médicas, 21(3), 606-618. https://tinyurl.com/y578pdm8

Sánchez-Álvarez, N., Extremera, N., Rey, L., Chang, E. C., \& Chang, O. D. (2020). Optimism and gratitude on suicide risk in Spanish adults: Evidence for doubling up or doubling down?. Journal of Clinical Psychology, 1-11- https://doi.org/10.1002/jclp.22962

Santamarina-Perez, P., Mendez, I., Singh, M. K., Berk, M., Picado, M., Font, E., Moreno, E., Martínez, E., Morer, A., Borràs, R., Cosi, A., \& Romero, S. (2020). Adapted Dialectical Behavior Therapy for Adolescents with a High Risk of Suicide in a Community Clinic: A Pragmatic Randomized Controlled Trial. Suicide Life Threat Behav, 50, 652-667. https://doi.org/10.1111/sltb.12612

Sarmiento, C., \& Villalobos, J. (2011). Predictores familiares y personales de la ideación suicida en adolescentes. Psicología y Salud, 21(1), 25. https://doi.org/10.25009/pys.v21i1.583

Schutte, N. S., Malouff, J. M., Thorsteinsson, E. B., Bhullar, N., \& Rooke, S. E. (2007). A metaanalytic investigation of the relationship between emotional intelligence and health. Personality and Individual Differences, 42(6), 921-933. https://doi.org/10.1016/ j.paid.2006.09.003

Siabato, E., \& Salamanca, Y. (2015). Factores asociados a ideación suicida en universitarios. Psychologia: Avances de la Disciplina, 9(1), 71-81. https://tinyurl.com/y4cm9ssh

Súarez, Y., Restrepo, D., \& Caballero, C. (2016). Ideación suicida y su relación con la inteligencia emocional en universitarios colombianos. Revista de la Universidad Industrial de Santander, Salud, 48(4), 470-478. https://doi.org/10.18273/revsal.v48n4-2016005

Villalobos, F. (2009). Situación de la conducta suicida en estudiantes de colegios y universidades de San Juan de Pasto, Colombia. Salud Mental, 31, 165-171. https://tinyurl.com/y5ogea8b 
Werbart Törnblom, A., Sorjonen, K., Runeson, B., y Rydelius, P.-A. (2020). Who Is at Risk of Dying Young from Suicide and Sudden Violent Death? Common and Specific Risk Factors among Children, Adolescents, and Young Adults. Suicide Life Threat Behav, 50, 757-777. https://doi.org/10.1111/sltb.12614

WHO. (2014). Preventing suicide: a global imperative. Geneve: World Health Organization. https://tinyurl.com/y6rr3q7o

WHO. (2018). National suicide prevention strategies: Progress, examples and indicators. Geneva: World Health Organization. https://tinyurl.com/y6c7mty8

Zadravec, N., Podlogar, T., Kerr, D., \& Leo, D. (2017). Community social support as a protective factor against suicide: A gender specific ecological study of 75 regions of 23 European countries. Health \& Place, 48, 40-46. https://doi.org/10.1016/j.healthplace.2017.09.004

\section{Para citar en APA}

Gómez Tabares, A. S., Núñez, C., Agudelo Osorio, M. P. y Caballo, V. E. (2020). Riesgo suicida y su relación con la inteligencia emocional y la autoestima en estudiantes universitarios Terapia Psicológica (En línea), 38(3), 403-426. https://doi.org/10.4067/S0718-48082020000 300403 